\title{
EXISTENCE OF POSITIVE SOLUTIONS FOR CERTAIN PARTIAL DIFFERENCE EQUATIONS
}

\author{
BINGGEN ZHANG AND QIUJU XING
}

Received 27 June 2005; Accepted 10 October 2005

We give some sufficient conditions for the existence of positive solutions of partial difference equation $a A_{m+1, n+1}+b A_{m, n+1}+c A_{m+1, n}-d A_{m, n}+P_{m, n} A_{m-k, n-l}=0$ by two different methods.

Copyright (c) 2006 B. Zhang and Q. Xing. This is an open access article distributed under the Creative Commons Attribution License, which permits unrestricted use, distribution, and reproduction in any medium, provided the original work is properly cited.

\section{Introduction}

In this paper, we consider the linear partial difference equation

$$
a A_{m+1, n+1}+b A_{m, n+1}+c A_{m+1, n}-d A_{m, n}+P_{m, n} A_{m-k, n-l}=0,
$$

where $P_{m, n}>0$ on $N_{0}^{2}, k, l \in N_{0}, N_{i}=\{i, i+1, \ldots\}$ and $i$ is an arbitrary integer. Throughout this paper, we assume that $a, b, c, d$ are positive constants.

A double sequence $\left\{A_{m, n}\right\}$ is said to be a solution of (1.1) if it satisfies (1.1) for $m \geq m_{0}$, $n \geq n_{0}$. A solution $\left\{A_{i, j}\right\}$ of $(1.1)$ is said to be eventually positive if $A_{i, j}>0$ for all large $i$ and $j$, and eventually negative if $A_{i, j}<0$ for all large $i$ and $j$. It is said to be oscillatory if it is neither eventually positive nor eventually negative.

The oscillation of (1.1) has been studied in [2]. In the following, we mainly consider the existence of positive solutions of (1.1) by two different methods.

\section{The method of fixed point}

The following lemma will be used to prove our main results in this section.

Lemma 2.1 [3] (Knaster's fixed point theorem). Let X be a partially ordered Banach space with ordering $\leq$. Let $M$ be a subset of $X$ with the following properties: the infimum of $M$ belongs to $M$ and every nonempty subset of $M$ has a supremum which belongs to $M$. Let 


\section{Existence of positive solutions for PDEs}

$T: M \rightarrow M$ be an increasing mapping, that is, $x \leq y$ implies $T x \leq T y$. Then $T$ has a fixed point in $M$.

Theorem 2.2. Assume that $a \geq d, b \geq d, c \geq d$, and one of the following three conditions holds.

(i) There exists a positive double sequence $\left\{\lambda_{m, n}\right\}$ such that for all sufficiently large $m, n$,

$$
\begin{aligned}
\frac{1}{d \lambda_{m, n}}\{ & \sum_{j=0}^{\infty} \sum_{i=0}^{\infty}(a-d) \lambda_{m+1+i, n+1+i+j}+\sum_{j=0}^{\infty} \sum_{i=1}^{\infty} b \lambda_{m+i, n+1+i+j} \\
& \left.+\sum_{j=0}^{\infty} \sum_{i=0}^{\infty} c \lambda_{m+1+i, n+i+j}+\sum_{j=0}^{\infty}(b-d) \lambda_{m, n+1+j}+\sum_{j=0}^{\infty} \sum_{i=0}^{\infty} P_{m+i, n+i+j} \lambda_{m-k+i, n-l+i+j}\right\} \leq 1 .
\end{aligned}
$$

(ii) There exists a positive double sequence $\left\{\lambda_{m, n}\right\}$ such that for all sufficiently large $m, n$,

$$
\begin{aligned}
\frac{1}{d \lambda_{m, n}}\{ & \sum_{j=0}^{\infty} \sum_{i=0}^{\infty}(a-d) \lambda_{m+1+i+j, n+1+i}+\sum_{j=0}^{\infty} \sum_{i=0}^{\infty} b \lambda_{m+i+j, n+1+i} \\
& \left.+\sum_{j=0}^{\infty} \sum_{i=1}^{\infty} c \lambda_{m+1+i+j, n+i}+\sum_{j=0}^{\infty}(c-d) \lambda_{m+1+j, n}+\sum_{j=0}^{\infty} \sum_{i=0}^{\infty} P_{m+i+j, n+i} \lambda_{m-k+i+j, n-l+i}\right\} \leq 1 .
\end{aligned}
$$

(iii) There exists a positive double sequence $\left\{\lambda_{m, n}\right\}$ such that for all sufficiently large $m, n$,

$$
\begin{aligned}
\frac{1}{d \lambda_{m, n}}\{ & \sum_{i=0}^{\infty} \sum_{j=0}^{\infty} a \lambda_{m+1+i, n+1+j}+\sum_{i=0}^{\infty} \sum_{j=0}^{\infty}(b-d) \lambda_{m+i, n+1+j} \\
& \left.+\sum_{i=0}^{\infty} \sum_{j=1}^{\infty} c \lambda_{m+1+i, n+j}+\sum_{i=0}^{\infty}(c-d) \lambda_{m+1+i, n}+\sum_{i=0}^{\infty} \sum_{j=0}^{\infty} P_{m+i, n+j} \lambda_{m-k+i, n-l+j}\right\} \leq 1 .
\end{aligned}
$$

Then (1.1) has an eventually positive solution $\left\{A_{m, n}\right\}$ which satisfies $0<A_{m, n} \leq \lambda_{m, n}$.

Proof. We only give the proof of (i), and the other cases are similar.

Let $X$ be the set of all real bounded double sequence $y=\left\{y_{m, n}\right\}_{m=m_{0}, n=n_{0}}^{\infty, \infty}$ with the norm $\|y\|=\sup _{m \geq m_{0}, n \geq n_{0}}\left|y_{m, n}\right|<\infty$. It is easy to prove that $X$ is a Banach space. We define a subset $\Omega$ of $X$ as follows:

$$
\Omega=\left\{y=\left\{y_{m, n}\right\} \in X: 0 \leq y_{m, n} \leq 1, m \geq m_{0}, n \geq n_{0}\right\}
$$

and define a partial order on $X$ in the usual way, that is,

$$
x, y \in X, \quad x \leq y \text { means that } x_{m, n} \leq y_{m, n} \text { for } m \geq m_{0}, n \geq n_{0} .
$$

It is easy to see that for any subset $S$ of $\Omega$, there exist inf $S$ and $\sup S$. We choose $m_{1}>m_{0}$, $n_{1}>n_{0}$ sufficiently large such that (i) holds. 
Set

$$
\begin{gathered}
D=N_{m_{0}} \times N_{n_{0}}, \quad D_{1}=N_{m_{1}} \times N_{n_{1}}, \\
D_{2}=\left(N_{m_{0}} \times N_{n_{1}}\right) \backslash D_{1}, \quad D_{3}=\left(N_{m_{1}} \times N_{n_{0}}\right) \backslash D_{1}, \\
D_{4}=D \backslash\left(D_{1} \cup D_{2} \cup D_{3}\right) .
\end{gathered}
$$

Clearly, $D=D_{1} \cup D_{2} \cup D_{3} \cup D_{4}$. Define a mapping $T: \Omega \rightarrow X$ as follows:

$$
T y_{m, n}=\left\{\begin{array}{rlrl}
\frac{1}{d \lambda_{m, n}}\left\{\sum_{j=0}^{\infty} \sum_{i=0}^{\infty}(a-d) \lambda_{m+1+i, n+1+i+j} y_{m+1+i, n+1+i+j}\right. & \\
& +\sum_{j=0}^{\infty} \sum_{i=1}^{\infty} b \lambda_{m+i, n+1+i+j} y_{m+i, n+1+i+j} & \\
& +\sum_{j=0}^{\infty} \sum_{i=0}^{\infty} c \lambda_{m+1+i, n+i+j} y_{m+1+i, n+i+j} & \\
& +\sum_{j=0}^{\infty}(b-d) \lambda_{m, n+1+j} y_{m, n+1+j} & \\
& \left.+\sum_{j=0}^{\infty} \sum_{i=0}^{\infty} P_{m+i, n+i+j} \lambda_{m-k+i, n-l+i+j} y_{m-k+i, n-l+i+j}\right\}, & (m, n) \in D_{1}, \\
\frac{n}{n_{1}} T y_{m_{1}, n}+\left(1-\frac{n}{n_{1}}\right), & (m, n) \in D_{2}, \\
\frac{m}{m_{1}} T y_{m, n_{1}}+\left(1-\frac{m}{m_{1}}\right), & (m, n) \in D_{3}, \\
\frac{m n}{m_{1} n_{1}} T y_{m_{1}, n_{1}}+\left(1-\frac{m n}{m_{1} n_{1}}\right), & (m, n) \in D_{4} .
\end{array}\right.
$$

From (2.7) and noting that $y_{m, n} \leq 1$, we have

$$
\begin{aligned}
0 \leq T y_{m, n} \leq \frac{1}{d \lambda_{m, n}}\left\{\sum_{j=0}^{\infty} \sum_{i=0}^{\infty}(a-d) \lambda_{m+1+i, n+1+i+j}+\sum_{j=0}^{\infty} \sum_{i=1}^{\infty} b \lambda_{m+i, n+1+i+j}\right. \\
+\sum_{j=0}^{\infty} \sum_{i=0}^{\infty} c \lambda_{m+1+i, n+i+j}+\sum_{j=0}^{\infty}(b-d) \lambda_{m, n+1+j} \\
\left.+\sum_{j=0}^{\infty} \sum_{i=0}^{\infty} P_{m+i, n+i+j} \lambda_{m-k+i, n-l+i+j}\right\} \leq 1, \quad \text { for }(m, n) \in D_{1}, \\
0 \leq T y_{m, n} \leq 1 \quad \text { for }(m, n) \in D_{2} \cup D_{3} \cup D_{4} .
\end{aligned}
$$




\section{Existence of positive solutions for PDEs}

Therefore, $T \Omega \subset \Omega$. Clearly, $T$ is nondecreasing. By Lemma 2.1, there is a $y \in \Omega$ such that $T y=y$, that is,

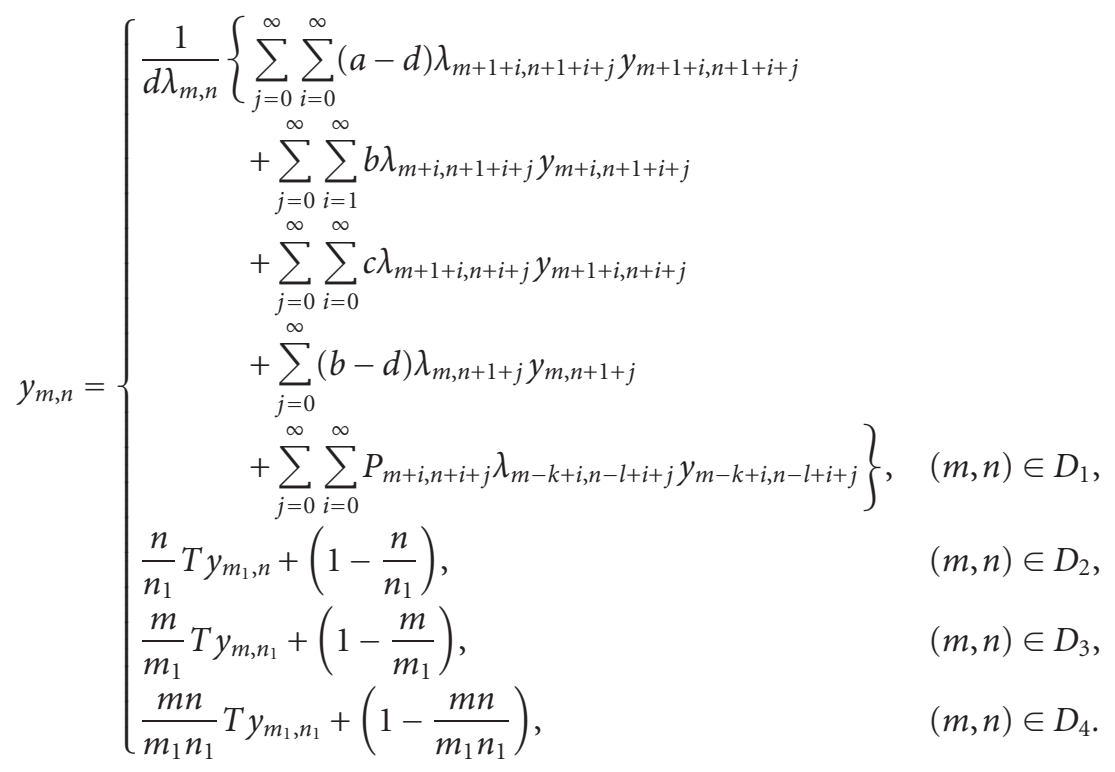

It is easy to see that $y_{m, n}>0$ for $(m, n) \in D_{2} \cup D_{3} \cup D_{4}$, and hence $y_{m, n}>0$ for all $(m, n) \in$ $D_{1}$. Set

$$
x_{m, n}=\lambda_{m, n} y_{m, n},
$$

then from (2.9) and (2.10), we have

$$
x_{m, n}=\left\{\begin{array}{rlrl}
\frac{1}{d}\left\{\sum_{j=0}^{\infty} \sum_{i=0}^{\infty}(a-d) x_{m+1+i, n+1+i+j}+\sum_{j=0}^{\infty} \sum_{i=1}^{\infty} b x_{m+i, n+1+i+j}\right. & \\
& +\sum_{j=0}^{\infty} \sum_{i=0}^{\infty} c x_{m+1+i, n+i+j}+\sum_{j=0}^{\infty}(b-d) x_{m, n+1+j} & \\
& \left.+\sum_{j=0}^{\infty} \sum_{i=0}^{\infty} P_{m+i, n+i+j} x_{m-k+i, n-l+i+j}\right\}, & (m, n) \in D_{1}, \\
\frac{n}{n_{1}} T y_{m_{1}, n}+\left(1-\frac{n}{n_{1}}\right), & (m, n) \in D_{2}, \\
\frac{m}{m_{1}} T y_{m, n_{1}}+\left(1-\frac{m}{m_{1}}\right), & (m, n) \in D_{3}, \\
\frac{m n}{m_{1} n_{1}} T y_{m_{1}, n_{1}}+\left(1-\frac{m n}{m_{1} n_{1}}\right), & (m, n) \in D_{4},
\end{array}\right.
$$

and so

$$
a x_{m+1, n+1}+b x_{m, n+1}+c x_{m+1, n}-d x_{m, n}+P_{m, n} x_{m-k, n-l}=0, \quad(m, n) \in D_{1},
$$

which implies that $x=\left\{x_{m, n}\right\}$ is a positive solution of (1.1). The proof is complete. 
Similarly, we can get the following conclusions according to variant relations of coefficients of (1.1).

Theorem 2.3. Assume that $a \geq d, b \geq d, c<d$, and one of the following three conditions holds.

(i) Equation (2.1) holds.

(ii) There exists a positive double sequence $\left\{\lambda_{m, n}\right\}$ such that for all sufficiently large $m, n$,

$$
\begin{aligned}
\frac{1}{d \lambda_{m, n}}\{ & \sum_{j=0}^{\infty} \sum_{i=1}^{\infty}\left(\frac{c}{d}\right)^{i} a \lambda_{m+1+i+j, n+1+j}+\sum_{j=0}^{\infty} \sum_{i=0}^{\infty}\left(\frac{c}{d}\right)^{i} b \lambda_{m+i+j, n+1+j} \\
& \left.+\sum_{j=0}^{\infty}(a-d) \lambda_{m+1+j, n+1+j}+\sum_{j=0}^{\infty} \sum_{i=0}^{\infty}\left(\frac{c}{d}\right)^{i} P_{m+i+j, n+j} \lambda_{m-k+i+j, n-l+j}\right\} \leq 1 .
\end{aligned}
$$

(iii) There exists a positive double sequence $\left\{\lambda_{m, n}\right\}$ such that for all sufficiently large $m, n$,

$$
\begin{aligned}
\frac{1}{d \lambda_{m, n}}\{ & \sum_{j=0}^{\infty} \sum_{i=0}^{\infty}\left(\frac{c}{d}\right)^{i} a \lambda_{m+1+i, n+1+j}+\sum_{j=0}^{\infty} \sum_{i=1}^{\infty}\left(\frac{c}{d}\right)^{i} b \lambda_{m+i, n+1+j} \\
& \left.+\sum_{j=0}^{\infty}(b-d) \lambda_{m, n+1+j}+\sum_{j=0}^{\infty} \sum_{i=0}^{\infty}\left(\frac{c}{d}\right)^{i} P_{m+i, n+j} \lambda_{m-k+i, n-l+j}\right\} \leq 1 .
\end{aligned}
$$

Then (1.1) has an eventually positive solution $\left\{A_{m, n}\right\}$ which satisfies $0<A_{m, n} \leq \lambda_{m, n}$.

Theorem 2.4. Assume that $a \geq d, b<d, c \geq d$, and one of the following three conditions holds.

(i) Equation (2.2) holds.

(ii) There exists a positive double sequence $\left\{\lambda_{m, n}\right\}$ such that for all sufficiently large $m, n$,

$$
\begin{aligned}
\frac{1}{d \lambda_{m, n}}\{ & \sum_{i=0}^{\infty} \sum_{j=1}^{\infty}\left(\frac{b}{d}\right)^{j} a \lambda_{m+1+i, n+1+i+j}+\sum_{i=0}^{\infty} \sum_{j=0}^{\infty}\left(\frac{b}{d}\right)^{j} c \lambda_{m+1+i, n+i+j} \\
& \left.+\sum_{i=0}^{\infty}(a-d) \lambda_{m+1+i, n+1+i}+\sum_{i=0}^{\infty} \sum_{j=0}^{\infty}\left(\frac{b}{d}\right)^{j} P_{m+i, n+i+j} \lambda_{m-k+i, n-l+i+j}\right\} \leq 1 .
\end{aligned}
$$

(iii) There exists a positive double sequence $\left\{\lambda_{m, n}\right\}$ such that for all sufficiently large $m, n$,

$$
\begin{aligned}
\frac{1}{d \lambda_{m, n}}\{ & \sum_{i=0}^{\infty} \sum_{j=0}^{\infty}\left(\frac{b}{d}\right)^{j} a \lambda_{m+1+i, n+1+j}+\sum_{i=0}^{\infty} \sum_{j=1}^{\infty}\left(\frac{b}{d}\right)^{j} c \lambda_{m+1+i, n+j} \\
& \left.+\sum_{i=0}^{\infty}(c-d) \lambda_{m+1+i, n}+\sum_{i=0}^{\infty} \sum_{j=0}^{\infty}\left(\frac{b}{d}\right)^{j} P_{m+i, n+j} \lambda_{m-k+i, n-l+j}\right\} \leq 1 .
\end{aligned}
$$

Then (1.1) has an eventually positive solution $\left\{A_{m, n}\right\}$ which satisfies $0<A_{m, n} \leq \lambda_{m, n}$. 
Theorem 2.5. Assume that $a \geq d, b<d, c<d$, and one of the following three conditions holds.

(i) Equation (2.13) holds.

(ii) Equation (2.15) holds.

(iii) There exists a positive double sequence $\left\{\lambda_{m, n}\right\}$ such that for all sufficiently large $m, n$,

$$
\begin{aligned}
\frac{1}{d \lambda_{m, n}}\{ & \sum_{i=0}^{\infty} \sum_{j=0}^{\infty}\left(\frac{c}{d}\right)^{i}\left(\frac{b}{d}\right)^{j} a \lambda_{m+1+i, n+1+j} \\
& \left.+\sum_{i=0}^{\infty} \sum_{j=1}^{\infty}\left(\frac{c}{d}\right)^{i}\left(\frac{b}{d}\right)^{j} c \lambda_{m+1+i, n+j}+\sum_{i=0}^{\infty} \sum_{j=0}^{\infty}\left(\frac{c}{d}\right)^{i}\left(\frac{b}{d}\right)^{j} P_{m+i, n+j} \lambda_{m-k+i, n-l+j}\right\} \leq 1 .
\end{aligned}
$$

Then (1.1) has an eventually positive solution $\left\{A_{m, n}\right\}$ which satisfies $0<A_{m, n} \leq \lambda_{m, n}$.

Theorem 2.6. Assume that $a<d, b \geq d, c \geq d$, and one of the following three conditions holds.

(i) There exists a positive double sequence $\left\{\lambda_{m, n}\right\}$ such that for all sufficiently large $m, n$,

$$
\begin{aligned}
\frac{1}{d \lambda_{m, n}}\{ & \sum_{j=0}^{\infty} \sum_{i=1}^{\infty}\left(\frac{a}{d}\right)^{i} b \lambda_{m+i, n+1+i+j}+\sum_{j=0}^{\infty} \sum_{i=0}^{\infty}\left(\frac{a}{d}\right)^{i} c \lambda_{m+1+i, n+i+j} \\
& \left.+\sum_{j=0}^{\infty}(b-d) \lambda_{m, n+1+j}+\sum_{j=0}^{\infty} \sum_{i=0}^{\infty}\left(\frac{a}{d}\right)^{i} P_{m+i, n+i+j} \lambda_{m-k+i, n-l+i+j}\right\} \leq 1 .
\end{aligned}
$$

(ii) There exists a positive double sequence $\left\{\lambda_{m, n}\right\}$ such that for all sufficiently large $m, n$,

$$
\begin{aligned}
\frac{1}{d \lambda_{m, n}}\left\{\sum_{j=0}^{\infty} \sum_{i=0}^{\infty}\left(\frac{a}{d}\right)^{i} b \lambda_{m+i+j, n+1+i}+\sum_{j=0}^{\infty} \sum_{i=1}^{\infty}\left(\frac{a}{d}\right)^{i} c \lambda_{m+1+i+j, n+i}\right. \\
\left.+\sum_{j=0}^{\infty}(c-d) \lambda_{m+1+j, n}+\sum_{j=0}^{\infty} \sum_{i=0}^{\infty}\left(\frac{a}{d}\right)^{i} P_{m+i+j, n+i} \lambda_{m-k+i+j, n-l+i}\right\} \leq 1 .
\end{aligned}
$$

(iii) Equation (2.3) holds.

Then (1.1) has an eventually positive solution $\left\{A_{m, n}\right\}$ which satisfies $0<A_{m, n} \leq \lambda_{m, n}$.

Theorem 2.7. Assume that $a<d, b \geq d, c<d$, and one of the following three conditions holds.

(i) Equation (2.18) holds.

(ii) Equation (2.14) holds. 
(iii) There exists a positive double sequence $\left\{\lambda_{m, n}\right\}$ such that for all sufficiently large $m, n$,

$$
\begin{aligned}
\frac{1}{d \lambda_{m, n}}\{ & \left\{\sum_{j=0}^{\infty} \sum_{i=0}^{\infty}\left(\frac{c}{d}\right)^{j}\left(\frac{a}{d}\right)^{i} b \lambda_{m+i+j, n+1+i}\right. \\
& \left.+\sum_{j=0}^{\infty} \sum_{i=1}^{\infty}\left(\frac{c}{d}\right)^{j}\left(\frac{a}{d}\right)^{i} c \lambda_{m+1+i+j, n+i}+\sum_{j=0}^{\infty} \sum_{i=0}^{\infty}\left(\frac{c}{d}\right)^{j}\left(\frac{a}{d}\right)^{i} P_{m+i+j, n+i} \lambda_{m-k+i+j, n-l+i}\right\} \leq 1 .
\end{aligned}
$$

Then (1.1) has an eventually positive solution $\left\{A_{m, n}\right\}$ which satisfies $0<A_{m, n} \leq \lambda_{m, n}$.

Theorem 2.8. Assume that $a<d, b<d, c \geq d$, and one of the following three conditions holds.

(i) Equation (2.19) holds.

(ii) Equation (2.16) holds.

(iii) There exists a positive double sequence $\left\{\lambda_{m, n}\right\}$ such that for all sufficiently large $m, n$,

$$
\begin{aligned}
\frac{1}{d \lambda_{m, n}}\{ & \sum_{j=0}^{\infty} \sum_{i=1}^{\infty}\left(\frac{b}{d}\right)^{j}\left(\frac{a}{d}\right)^{i} b \lambda_{m+i, n+1+i+j} \\
& \left.+\sum_{j=0}^{\infty} \sum_{i=0}^{\infty}\left(\frac{b}{d}\right)^{j}\left(\frac{a}{d}\right)^{i} c \lambda_{m+1+i, n+i+j}+\sum_{j=0}^{\infty} \sum_{i=0}^{\infty}\left(\frac{b}{d}\right)^{j}\left(\frac{a}{d}\right)^{i} P_{m+i, n+i+j} \lambda_{m-k+i, n-l+i+j}\right\} \leq 1 .
\end{aligned}
$$

Then (1.1) has an eventually positive solution $\left\{A_{m, n}\right\}$ which satisfies $0<A_{m, n} \leq \lambda_{m, n}$.

Theorem 2.9. Assume that $a<d, b<d, c<d$, and one of the following three conditions holds.

(i) Equation (2.20) holds.

(ii) Equation (2.21) holds.

(iii) Equation (2.17) holds.

Then (1.1) has an eventually positive solution $\left\{A_{m, n}\right\}$ which satisfies $0<A_{m, n} \leq \lambda_{m, n}$.

Example 2.10. Consider the equation

$$
\frac{1}{4} A_{m+1, n+1}+\frac{1}{4} A_{m, n+1}+\frac{1}{4} A_{m+1, n}-\frac{19}{2} A_{m, n}+120 A_{m-2, n-2}=0 .
$$

We can find that $a<d, b<d, c<d$, and $\lambda_{m, n}=5^{m+n}$ satisfies (2.21). By Theorem 2.9, (2.22) has a positive solution $\left\{A_{m, n}\right\}$ which satisfies $0<A_{m, n} \leq \lambda_{m, n}$. In fact, $A_{m, n}=2^{m+n}$ is such a solution of (2.22).

\section{The method of construction}

Above all, we define several sets of vertices: $\Omega=\{(i, j): i, j$ are integers and $i \geq-k, j \geq$ $-l\}$ where $k$ and $l$ are the same ones used in (1.1), $\Omega^{+}=\{(i, j) \in \Omega: i, j \geq 0\}$ and $\Omega^{*}=$ $\{(i, j) \in \Omega: i \geq 0$ or $j \geq 0\}$.

Given a vertex $z=(i, j) \in \Omega$, the two adjacent vertices $(i+1, j)$ and $(i, j+1)$ will be denoted by $z_{R}$ and $z_{T}$, respectively, and the vertex $(i+1, j+1)$ will be denoted by $z_{R T}$. 
Furthermore, we may also introduce an ordering for these vertices as follows: let $w=$ $(m, n)$ and $z=(i, j)$ be vertices in $\Omega$, we say that $w$ precedes $z$, denoted by $w \leq z$ if $m \leq i$ and $n \leq j$. As usual, we will denote the vertex $(0,0)$ by 0 . The vertex $(k, l)$ and $(m-i, n-$ $j)$ will be denoted by $\delta$ and $w-z$ respectively.

For each pair of vertices $w=(m, n) \in \Omega^{+}$and $z=(i, j) \in \Omega$, we associate a real value $f(w, z)$. Thus we have a function $f$ defined on $\Omega^{+} \times \Omega$, and we say that $f$ is positive if $f(w, z)>0$ for all $w \in \Omega^{+}$and $z \in \Omega$.

Consider the equation

$$
a f\left(w, w_{R T}\right)+b f\left(w, w_{T}\right)+c f\left(w, w_{R}\right)-d f(w, w)+P(w) f(w, w-\delta)=0, \quad w \in \Omega^{+},
$$

where $P(w)$ is a positive function defined at each vertex in $\Omega^{+}$and $a, b, c, d$ are the same ones used in (1.1).

LEMma 3.1. If (3.1) has a positive solution $f(w, w)$, then (1.1) has an eventually positive solution.

Proof. Suppose (3.1) has a positive solution $f(w, w)$, then we can define a sequence $\left\{A_{i, j}\right\}$ by $A_{i, j}=f(w, z)$, and thus $A_{m, n}=f(w, w)$ is an eventually positive solution of (1.1).

In the following, we consider two cases: (1) $a=0$; (2) $a \neq 0$.

Case 1. $a=0$.

For this case, we will rely on the following nonlinear equation

$$
\phi_{m, n}=P_{m, n}\left(\prod_{s=m-k}^{m-1} \frac{b+c}{d-\phi_{s, n}}\right)\left(\prod_{t=n-l}^{n-1} \frac{b+c}{d-\phi_{m, t}}\right), \quad m, n \geq 0
$$

where $k, l$ and $b, c, d, P_{m, n}$ are the same ones used in (1.1). Here and in the sequel, we will adopt the convention that an empty product equals one.

Note first that in order for a double sequence $\left\{\phi_{m, n}\right\}$ to satisfy (3.2), it is only necessary to define $\phi_{m, n}$ for $(m, n) \in \Omega^{*}$. Furthermore, we may show that if $\left\{\phi_{m, n}\right\}$ is a double sequence strictly bounded between 0 and $d$ for $(m, n) \in \Omega^{*}$ and satisfies (3.2), then $f(w, w)$, defined by

$$
f(w, z)=\left(\prod_{s=-k}^{i-1} \frac{d-\phi_{s, n}}{b+c}\right)\left(\prod_{t=-l}^{j-1} \frac{d-\phi_{m, t}}{b+c}\right)
$$

for each pair of vertices $w=(m, n) \in \Omega^{+}$and $z=(i, j) \in \Omega$, is clearly positive and will satisfy (3.1). 
Indeed, for any $w=(m, n) \in \Omega^{+}$,

$$
\begin{aligned}
b f\left(w, w_{T}\right) & +c f\left(w, w_{R}\right)-d f(w, w) \\
= & b\left(\prod_{s=-k}^{m-1} \frac{d-\phi_{s, n}}{b+c}\right)\left(\prod_{t=-l}^{n} \frac{d-\phi_{m, t}}{b+c}\right)+c\left(\prod_{s=-k}^{m} \frac{d-\phi_{s, n}}{b+c}\right)\left(\prod_{t=-l}^{n-1} \frac{d-\phi_{m, t}}{b+c}\right) \\
& -d\left(\prod_{s=-k}^{m-1} \frac{d-\phi_{s, n}}{b+c}\right)\left(\prod_{t=-l}^{n-1} \frac{d-\phi_{m, t}}{b+c}\right)=-\phi_{m, n}\left(\prod_{s=-k}^{m-1} \frac{d-\phi_{s, n}}{b+c}\right)\left(\prod_{t=-l}^{n-1} \frac{d-\phi_{m, t}}{b+c}\right),
\end{aligned}
$$

$P(w) f(w, w-\delta)$

$$
\begin{aligned}
& =P(w)\left(\prod_{s=-k}^{m-k-1} \frac{d-\phi_{s, n}}{b+c}\right)\left(\prod_{t=-l}^{n-l-1} \frac{d-\phi_{m, t}}{b+c}\right) \\
& =P(w)\left(\prod_{s=m-k}^{m-1} \frac{b+c}{d-\phi_{s, n}}\right)\left(\prod_{t=n-l}^{n-1} \frac{b+c}{d-\phi_{m, t}}\right)\left(\prod_{s=-k}^{m-1} \frac{d-\phi_{s, n}}{b+c}\right)\left(\prod_{t=-l}^{n-1} \frac{d-\phi_{m, t}}{b+c}\right) \\
& =\phi_{m, n}\left(\prod_{s=-k}^{m-1} \frac{d-\phi_{s, n}}{b+c}\right)\left(\prod_{t=-l}^{n-1} \frac{d-\phi_{m, t}}{b+c}\right)
\end{aligned}
$$

as required, and thus we get the following conclusion.

Theorem 3.2. Suppose there is a double sequence $\phi=\left\{\phi_{m, n}\right\}$ which satisfies (3.2) and $0<$ $\phi_{m, n}<d$ for $(m, n) \in \Omega^{*}$, then the function $f: \Omega^{+} \times \Omega \rightarrow R$ defined by (3.3) is a positive solution of (3.1).

The existence of $\left\{\phi_{m, n}\right\}$ can be seen in [1].

According to the analysis in [1], we can get the following conclusion similarly.

Corollary 3.3. Suppose either (i) $k=l=0, P_{m, n} \leq \lambda<d$; or (ii) $k+l>0$,

$$
P_{m, n} \leq \frac{d^{k+l+1}(k+l)^{k+l}}{(b+c)^{k+l}(k+l+1)^{k+l+1}},
$$

then there is a positive solution of (1.1).

Remark 3.4. In [2, Corollary 2.2], the authors give an oscillation criterion for (1.1) as follows: if

$$
\liminf _{m, n \rightarrow \infty} P_{m, n}=P>\frac{d^{k+l+1}(k+l)^{k+l}}{b^{k} c^{l}(1+k+l)^{1+k+l}}
$$

then every solution of (1.1) is oscillatory.

We can find that

$$
\frac{d^{k+l+1}(k+l)^{k+l}}{(b+c)^{k+l}(k+l+1)^{k+l+1}} \leq \frac{d^{k+l+1}(k+l)^{k+l}}{b^{k} c^{l}(1+k+l)^{1+k+l}}
$$


for $a=0$. Thus there is a gap between the oscillation condition (3.6) and condition (3.5) for the existence of positive solutions for the case $a=0$ in (1.1). How do we fill this gap is an open problem.

Case 2. $a \neq 0$.

For this case, we will rely on the nonlinear equation

$$
\begin{aligned}
\phi_{m, n}= & P_{m, n}\left(\prod_{s=m-k}^{m-1} \frac{\left[2 d a+c\left(b+c+\sqrt{(b+c)^{2}+4 a d}\right)\right]\left(b+c+\sqrt{(b+c)^{2}+4 a d}\right)}{2 d\left[2 d a+c\left(b+c+\sqrt{(b+c)^{2}+4 a d}\right)\right]-\left(b+c+\sqrt{(b+c)^{2}+4 a d}\right)^{2} \phi_{s, n}}\right) \\
& \times\left(\frac{b+c+\sqrt{(b+c)^{2}+4 a d}}{2 d}\right)^{l}
\end{aligned}
$$

or

$$
\begin{aligned}
\phi_{m, n}= & P_{m, n}\left(\frac{b+c+\sqrt{(b+c)^{2}+4 a d}}{2 d}\right)^{k} \\
& \times\left(\prod_{t=n-l}^{n-1} \frac{\left[2 d a+b\left(b+c+\sqrt{(b+c)^{2}+4 a d}\right)\right]\left(b+c+\sqrt{(b+c)^{2}+4 a d}\right)}{2 d\left[2 d a+b\left(b+c+\sqrt{(b+c)^{2}+4 a d}\right)\right]-\left(b+c+\sqrt{(b+c)^{2}+4 a d}\right)^{2} \phi_{m, t}}\right),
\end{aligned}
$$

where $k, l$ and $a, b, c, d, P_{m, n}$ are the same ones used in (1.1) and we can also define $f(w, z)$ as follows:

$$
\begin{aligned}
f(w, z)= & \left(\prod_{s=-k}^{i-1} \frac{2 d\left[2 d a+c\left(b+c+\sqrt{(b+c)^{2}+4 a d}\right)\right]-\left(b+c+\sqrt{(b+c)^{2}+4 a d}\right)^{2} \phi_{s, n}}{\left[2 d a+c\left(b+c+\sqrt{(b+c)^{2}+4 a d}\right)\right]\left(b+c+\sqrt{(b+c)^{2}+4 a d}\right)}\right) \\
& \times\left(\frac{2 d}{b+c+\sqrt{(b+c)^{2}+4 a d}}\right)^{j+l}
\end{aligned}
$$

or

$$
\begin{aligned}
f(w, z)= & \left(\frac{2 d}{b+c+\sqrt{(b+c)^{2}+4 a d}}\right)^{i+k} \\
& \times\left(\prod_{t=-l}^{j-1} \frac{2 d\left[2 d a+b\left(b+c+\sqrt{(b+c)^{2}+4 a d}\right)\right]-\left(b+c+\sqrt{(b+c)^{2}+4 a d}\right)^{2} \phi_{s, n}}{\left[2 d a+b\left(b+c+\sqrt{(b+c)^{2}+4 a d}\right)\right]\left(b+c+\sqrt{(b+c)^{2}+4 a d}\right)}\right)
\end{aligned}
$$

for $0<\phi_{m, n}<\left(2 d\left[2 d a+c\left(b+c+\sqrt{(b+c)^{2}+4 a d}\right)\right]\right) /\left(b+c+\sqrt{(b+c)^{2}+4 a d}\right)^{2}$ or $\left(2 d\left[2 d a+b\left(b+c+\sqrt{(b+c)^{2}+4 a d}\right)\right]\right) /\left(b+c+\sqrt{(b+c)^{2}+4 a d}\right)^{2}$ for $(m, n) \in \Omega^{*}$, and the following conclusion is similar to Theorem 3.2. 
Theorem 3.5. Suppose there is a double sequence $\phi=\left\{\phi_{m, n}\right\}$ which satisfies (3.8) (or (3.9)) and

$$
0<\phi_{m, n}<\frac{2 d\left[2 d a+c\left(b+c+\sqrt{(b+c)^{2}+4 a d}\right)\right]}{\left(b+c+\sqrt{(b+c)^{2}+4 a d}\right)^{2}}
$$

or

$$
\left(\frac{2 d\left[2 d a+b\left(b+c+\sqrt{(b+c)^{2}+4 a d}\right)\right]}{\left(b+c+\sqrt{(b+c)^{2}+4 a d}\right)^{2}}\right)
$$

for $(m, n) \in \Omega^{*}$, then the function $f: \Omega^{+} \times \Omega \rightarrow R$ defined by (3.10) (or (3.11)) is a positive solution of (3.1).

Corollary 3.6. Suppose either (i) $k=l=0$,

$$
\begin{gathered}
P_{m, n} \leq \lambda<\frac{2 d\left[2 d a+c\left(b+c+\sqrt{(b+c)^{2}+4 a d}\right)\right]}{\left(b+c+\sqrt{(b+c)^{2}+4 a d}\right)^{2}} \\
\quad \text { or }\left(\frac{2 d\left[2 d a+b\left(b+c+\sqrt{(b+c)^{2}+4 a d}\right)\right]}{\left(b+c+\sqrt{(b+c)^{2}+4 a d}\right)^{2}}\right) ;
\end{gathered}
$$

or (ii) $k+l>0$,

$$
P_{m, n} \leq \frac{k^{k}}{(1+k)^{1+k}} \frac{(2 d)^{k+l+1}\left[2 a d+c\left(b+c+\sqrt{(b+c)^{2}+4 a d}\right)\right]}{\left(b+c+\sqrt{(b+c)^{2}+4 a d}\right)^{k+l+2}}
$$

or

$$
P_{m, n} \leq \frac{l^{l}}{(1+l)^{1+l}} \frac{(2 d)^{k+l+1}\left[2 a d+b\left(b+c+\sqrt{(b+c)^{2}+4 a d}\right)\right]}{\left(b+c+\sqrt{(b+c)^{2}+4 a d}\right)^{k+l+2}},
$$

then there is a positive solution of (3.1).

Remark 3.7. In [2, Corollary 2.1], the authors give an oscillation criterion for (1.1) as follows: if

$$
\liminf _{m, n \rightarrow \infty}=P>d^{k+1}\left(\left(a+\frac{2 b c}{d}\right)^{l} b^{k-l}\right)^{-1} \frac{k^{k}}{(1+k)^{1+k}}, \quad \text { for } k \geq l
$$

or

$$
\liminf _{m, n \rightarrow \infty}=P>d^{l+1}\left(\left(a+\frac{2 b c}{d}\right)^{k} c^{l-k}\right)^{-1} \frac{l^{l}}{(1+l)^{1+l}}, \quad \text { for } l>k
$$

then every solution of (1.1) is oscillatory. 
We can also find that for $k \geq l$,

$$
\begin{gathered}
\frac{k^{k}}{(1+k)^{1+k}} \frac{(2 d)^{k+l+1}\left[2 a d+c\left(b+c+\sqrt{(b+c)^{2}+4 a d}\right)\right]}{\left(b+c+\sqrt{(b+c)^{2}+4 a d}\right)^{k+l+2}} \\
\leq d^{k+1}\left(\left(a+\frac{2 b c}{d}\right)^{l} b^{k-l}\right)^{-1} \frac{k^{k}}{(1+k)^{1+k}},
\end{gathered}
$$

and for $l>k$,

$$
\begin{gathered}
\frac{l^{l}}{(1+l)^{1+l}} \frac{(2 d)^{k+l+1}\left[2 a d+b\left(b+c+\sqrt{(b+c)^{2}+4 a d}\right)\right]}{\left(b+c+\sqrt{(b+c)^{2}+4 a d}\right)^{k+l+2}} \\
\leq d^{l+1}\left(\left(a+\frac{2 b c}{d}\right)^{k} c^{k-l}\right)^{-1} \frac{l^{l}}{(1+l)^{1+l}} .
\end{gathered}
$$

So in the case $a \neq 0$, there is also a gap between the oscillation condition and the condition for the existence of positive solutions of (1.1) in Corollary 3.6. Filling this gap is an open problem.

Example 3.8. Consider the equation

$$
3 f\left(w, w_{T}\right)+5 f\left(w, w_{R}\right)-6 f(w, w)+\left(6-e^{-(m+n)}\right) 8^{-4} e^{6-4(m+n)} f(w, w-\delta)=0,
$$

where $\delta=(2,2)$. Here we can find that $\phi_{m, n}=6-e^{-(m+n)}$ satisfies (3.2). Therefore, by Theorem 3.2, (3.21) has a positive solution. In fact, we can construct a solution of (3.21) by (3.3). Hence, by Lemma 3.1, the partial difference equation

$$
3 A_{m, n+1}+5 A_{m+1, n}-6 A_{m, n}+\left(6-e^{-(m+n)}\right) 8^{-4} e^{6-4(m+n)} A_{m-2, n-2}=0
$$

has a positive solution.

\section{References}

[1] S. S. Cheng, S. T. Liu, and B. Zhang, Positive flows of an infinite network, Communications in Applied Analysis 1 (1997), no. 1, 83-90.

[2] S. K. Choi and B. Zhang, Oscillation of certain partial difference equations, Discrete Dynamics in Nature and Society 2 (1998), no. 4, 257-265.

[3] L. H. Erbe, Q. K. Kong, and B. Zhang, Oscillation Theory for Functional Differential Equations, Monographs and Textbooks in Pure and Applied Mathematics, vol. 190, Marcel Dekker, New York, 1995.

Binggen Zhang: Department of Mathematics, Ocean University of China, Qingdao 266071, China E-mail address: bgzhang@public.qd.sd.cn

Qiuju Xing: Department of Mathematics and Physics, Xi'an Institute of Technology,

Xi' an 710032, China

E-mail address: xqj_001@163.com 


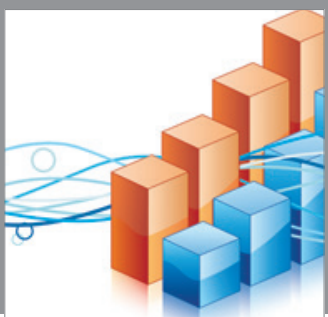

Advances in

Operations Research

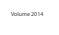

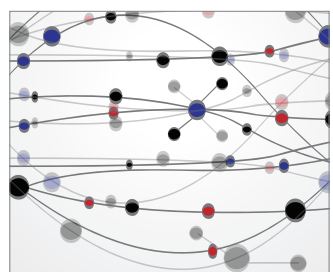

\section{The Scientific} World Journal
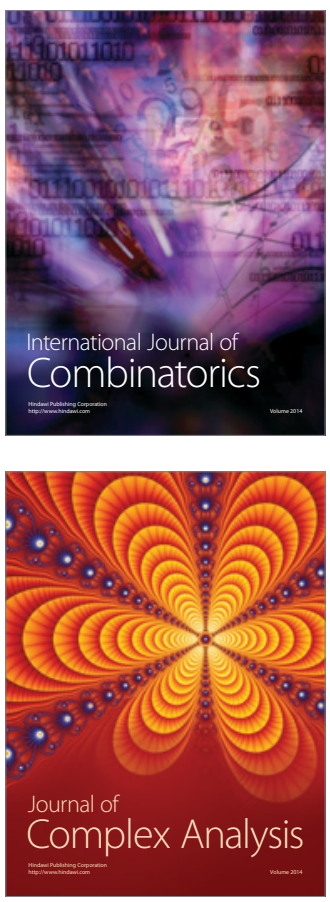

International Journal of

Mathematics and

Mathematical

Sciences
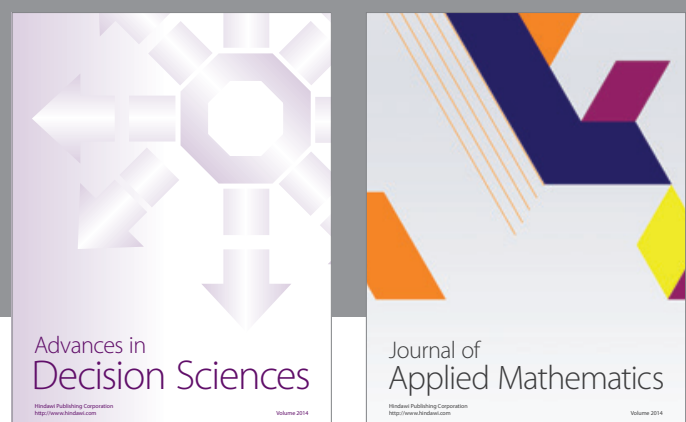

Journal of

Applied Mathematics
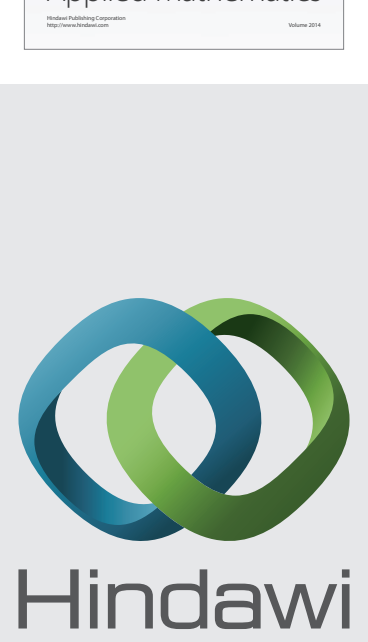

Submit your manuscripts at http://www.hindawi.com
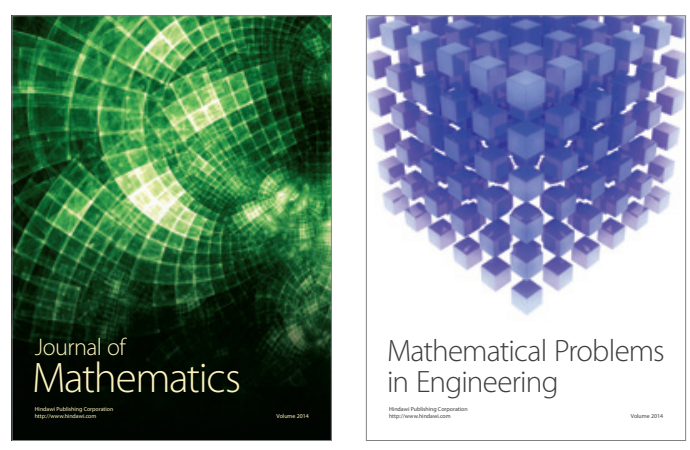

Mathematical Problems in Engineering
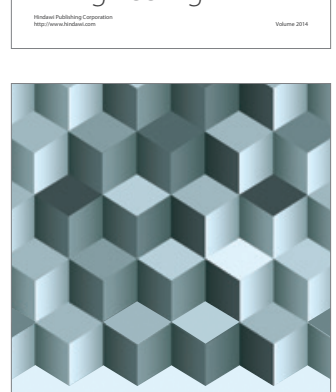

Journal of

Function Spaces
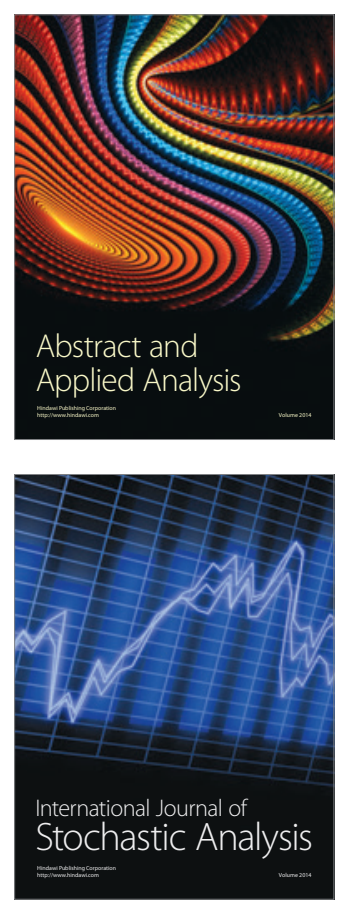

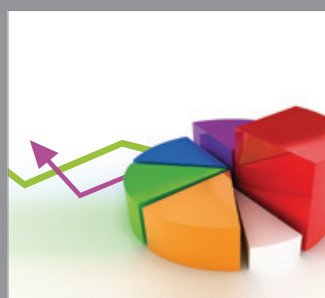

ournal of

Probability and Statistics

Promensencen
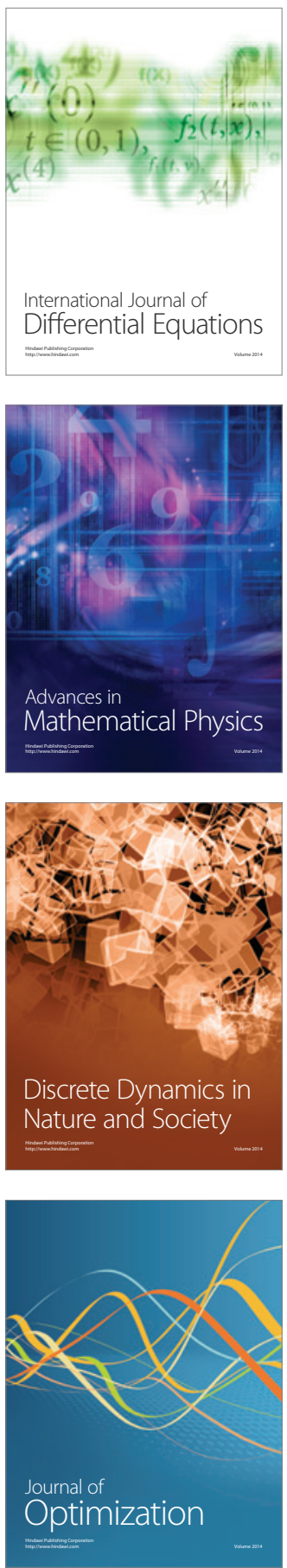